\title{
Yoğunluk Fonksiyoneli Teorisi ile LiAISi'un Basınç Altında Elektronik ve Titreşim Özellikleri
}

\author{
Sinem Erden Gülebağlan ${ }^{1^{*}}$ \\ ${ }^{1}$ Van Yüzüncüyıl Üniversitesi, Van Meslek Yüksekokulu Elektrik ve Enerji Bölümü, Van, Türkiye, (ORCID: 0000-0001-9446-2211)
}

(Ilk Geliş Tarihi 1 Aralık 2019 ve Kabul Tarihi 31 Aralık 2019)

(DOI: 10.31590/ejosat.629144)

ATIF/REFERENCE: Erden Gülebağlan, S. (2019). Yoğunluk Fonksiyoneli Teorisi ile LiAlSi’un Basınç Altında Elektronik ve Titreşim Özellikleri. Avrupa Bilim ve Teknoloji Dergisi, (17), 1340-1346.

$\ddot{O} \mathbf{z}$

Li içerikli malzemeler teknolojinin gelişmesinde önemli yere sahiptirler. Bu nedenle Li içerikli kristallerin yapısal elektronik ve dinamik özelliklerinin bilinmesi önemlidir. LiAlSi kristalinin yapısal, elektronik ve örgü dinamik özellikleri, ab-initio psödopotensiyel metodu ve Genel Gradyent Yaklaşımı ile doğrusal bir tepki ile gerçekleştirilmiştir. LiAlSi kristali Zincblende yapıda olup uzay grubu

$F \overline{4} 3 m$ 'dir. LiAlSi kristalinin örgü parametresi 6.0306 Å olarak bulunmuştur. Hesaplanan örgü parametresi, yığın modulü ve yığın modülünün birinci dereceden basınca göre türevi, deneysel ve diğer teorik hesaplamalar ile uyumludur. Elektronik band yapısı ve fonon dağılım eğrisi, Quantum Espresso programı kullanılarak analiz edilmiştir. Fonon dağılım eğrisi ve fonon durumların yoğunluğu, yoğunluk fonksiyonel pertürbasyon teorisi ile hesaplanmıştır. Fonon frekans değerleri pozitif olduğundan LiAlSi kristali kararlı yapıdadır. Daha sonra $\mathrm{P}=8.892 \mathrm{GPa}$ basınç altında elektronik band yapısı ve fonon dağılım eğrisi incelenmiştir. $\mathrm{P}=0.0 \mathrm{GPa}$ basınçta LiAlSi kristali yariletken özelliği gösterirken $\mathrm{P}=8.892 \mathrm{GPa}$ basınç uygulandığında iletken özelliği gösterdiği ortaya konmuştur. Bu basınç değerinde de fonon frekans değerleri pozitiftir. Ayrıca iki farklı basınç altında ( $\mathrm{P}=0.0 \mathrm{GPa}$ ve $\mathrm{P}=8.892 \mathrm{GPa}) \quad \Gamma, \mathrm{X}$ ve $\mathrm{L}$ yüksek simetri noktalarındaki enine, boyuna akustik ve enine, boyuna optik mod değerleri listelenmiştir. Bu çalışmanın sonuçlarının literatüre katkı sağlayacağı düşünülmektedir.

Anahtar Kelimeler: LiAlSi, Elektronik özellikler, Titreşim özellikleri, Yoğunluk Fonksiyoneli Teorisi.

\section{Electronic and Vibrational Properties of LiAISi under Pressure: A Density Functional Theory}

\begin{abstract}
Li containing materials have an important place in the development of technology. Therefore, knowledge of the structural and dynamic properties of electronic Li-containing crystals is important. The structural, electronic and lattice dynamic properties of LiAlSi crystal was performed by an ab-initio pseudopotential method and a linear response with the General Gradient Approximation.

LiAlSi crystal is in Zincblende structure and the space group is $F \overline{4} 3 m$. The lattice parameter of LiAlSi crystal was $6.0306 \AA$. The computed lattice parameter, bulk modulus and first-order derivation with respect to pressure of the bulk modulus agree well with the experimental and other theoretical calculations. The electronic band structure and phonon dispersion curve were analyzed by using the Quantum Espresso program. Phonon dispersion curve and phonon density of states were calculated by a density functional perturbation theory. Since the phonon frequency values are positive, the LiAlSi crystal is stable. Then, electronic band structure and phonon distribution curve under $\mathrm{P}=8.892 \mathrm{GPa}$ pressure were examined. LiAlSi crystal showed semiconductor properties at $\mathrm{P}=0.0$ GPa pressure, whereas $\mathrm{P}=8.892 \mathrm{GPa}$ pressure showed semi-metal properties. At this pressure, the phonon frequency values are also positive. In addition, transverse, longitudinal acoustic and transverse, longitudinal optical mode values at high symmetry points $\Gamma$, $\mathrm{X}$ and $\mathrm{L}$ under two different pressures $(\mathrm{P}=0.0 \mathrm{GPa}$ and $\mathrm{P}=8.892 \mathrm{GPa})$ are listed. It is thought that the results of this study will contribute to the literature.
\end{abstract}

Keywords: LiAlSi, Elektronic properties, Vibrational properties, Density Functional Theory.

\footnotetext{
* Sorumlu Yazar: Van Yüzüncüyıl Üniversitesi, Van Meslek Yüksekokulu Elektrik ve Enerji Bölümü, Van, Türkiye, ORCID: 0000-0001-9446-2211, sinemerdengulebaglan@yyu.edu.tr
} 


\section{Giriş}

Half- Heusler bileşikler termoelektrik, spintronik, optoelektronik malzeme üretiminde umut verici malzemelerdir (Kandpal ve diğ., 2006). Bu bileşikler ayarlanabilir enerji band aralıkları ve farklı elektronegatiflik özelliklerine sahiplerdir. Lityum orta derecedeki elektronegatif elementler ile kolayca bileşik oluşturmaktadırlar. Lityum tabanlı Half-Heusler bileşikleri geniş direkt ve indirekt band aralıklarına sahiptirler. Half-Heusler yapısı Li-dolu Zincblende yapısı olarak görülebilir (Casper ve diğ., 2012).

LiAlSi kristali için ilk araştırma Boom ve arkadaşları (1949) tarafından gerçekleştirilmiştir. Tillard ve arkadaşları (2005) LityumAlüminyum Silikondan oluşan en az dört bileşiğin var olduğunu ortaya koymuş ve bu bileşiklerin kristal yapılarını X-1şını analizinden yararlanarak incelemişlerdir. Tillard ve arkadaşları kristal yapılarını belirledikleri $\mathrm{LiAlSi}, \mathrm{Li}_{5} \mathrm{AlSi}_{2}$ ve $\mathrm{Li}_{9} \mathrm{AlSi}_{3}$ kristallerini kullanılarak Li/ $\mathrm{Li}_{x} \mathrm{Al}_{y} \mathrm{Si}_{3}$ elektrokimyasal hücrelerin galvonostik döngüsünden elde edilen sonuçları bildirmişlerdir. Ayrıca Yoğunluk Fonksiyoneli Teorisi ile bu bileşiklerin enerji stabilitesini ve elektronik yapılarını incelemişlerdir. Barth ve arkadaşları (2010) I-III-IV tabanlı LiAlSi ve LiAlGe Half- Heusler bileşiklerini deneysel olarak çalşarak bu bileşiklerin termoelektrik parametrelerini araştırmışlardır. 400 Kelvin de LiAlSi için Seeback katsayısını $160 \mathrm{VK}^{-1}$ olarak bulmuşlardır. Nowotny ve arakadaşları (1960) X-1şııı kırınımı yöntemiyle LiAlSi kristali örgü parametresinin $\mathrm{a}_{\mathrm{LiAlS}}=5.94 \AA$ ve uzay grubunun $F \overline{4} 3 m$ olduğunu bulmuşlardır. Spina ve arkadaşları (2003) LiAlSi, Li $\mathrm{Al}_{3} \mathrm{Si}_{4}, \mathrm{Li}_{18} \mathrm{Al}_{2} \mathrm{Si}_{6}, \mathrm{Li}_{15} \mathrm{Al}_{3} \mathrm{Si}_{6}$ yapılarını X 1şını kırınımı yöntemiyle incelemişlerdir. LiAlSi için Schuster ve arkadaşları (1976) da deneysel çalışma gerçekleştirmiş olup örgü parametresini $5.93 \AA$ olarak bulup, atomların koordinatlarını Li (1/2 1/2 1/2 ), $\mathrm{Al}(000$ ), Si (1/4 1/4 1/4 ) olarak tepit etmişlerdir. Bu atomik koordinatlar ile oluşturulmuş LiAıSi yapısının prototipi Şekil 1 de çizilmiştir. Hesaplamalar bu atomik koordinatlar kullanılarak gerçekleştirilmiş̧ir.

\section{Materyal ve Metot}

Yoğunluk Fonksiyoneli Teorisinin Genel Gradyent Yaklaşımı kullanılarak LiAlSi kristali için yapısal, elektronik ve dinamik özellikler incelenmiştir. Bu hesaplamalar için Quantum Espresso (Baroni ve diğ.,) paket programı kullanılmıştır. Değiş tokuş korelasyon enerjisi Genel Gradyent Yaklaşımı (Perdew ve diğ., 1996) içinde Perdev-Burke-Ernzerhof parametreleştirmesi kullanılarak tanımlanmıştır. Dalga fonksiyonları 80 Ryd'lik kesme enerjisi ile düzlem dalga temelinde genişletilmiştir. Şekil 2'de farklı kesme enerjilerine karşıllk gelen toplam enerji değerleri gösterilmiştir. Tablo 1 de de 20, 40, 60, 80 ve 100 Ryd kesme enerjisi değerlerine karşlık gelen toplam enerji değerleri listelenmiştir. Elektronik yük yoğunluğu için kesme enerjisi 320 Ryd olarak alınmıştır.

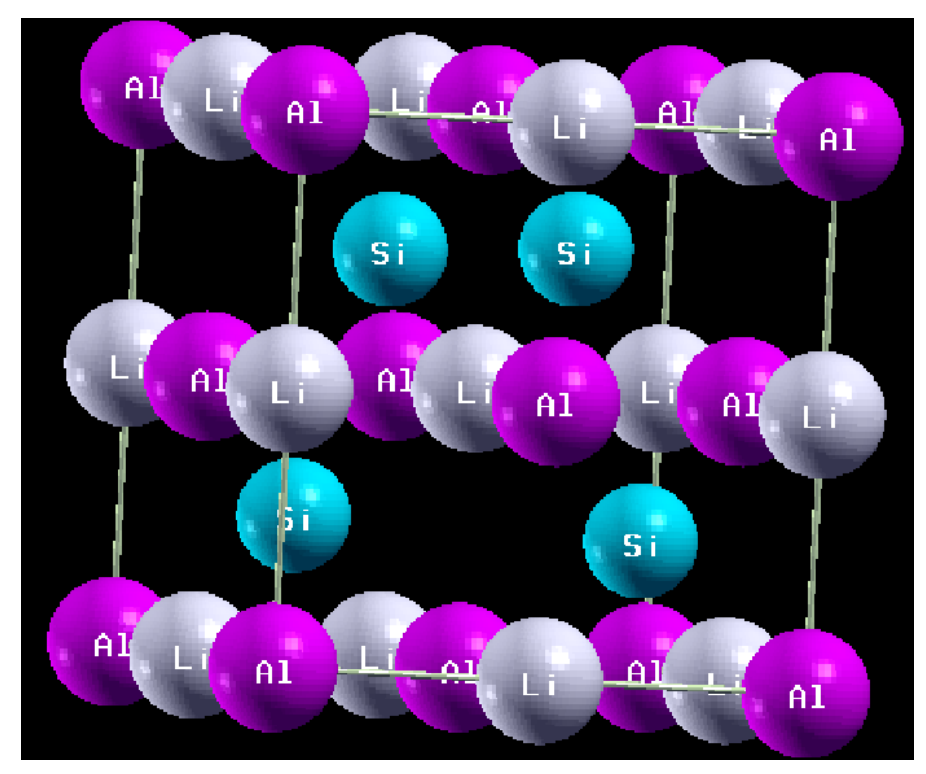

Şekil 1. LiAlSi kristalinin prototipi. 


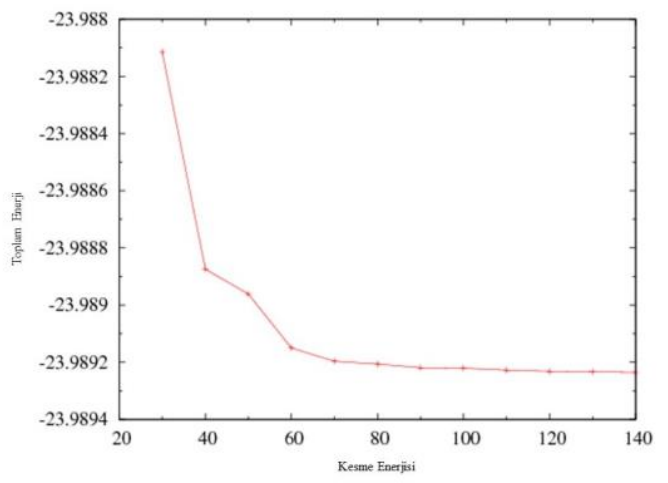

Şekil 2. LiAlSi kristali için kesme enerjisine karşılık gelen toplam enerji değeri.

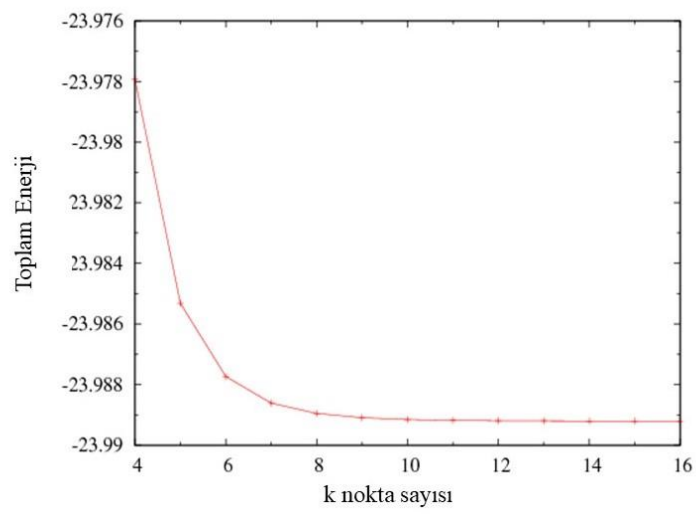

Şekil 3. LiAlSi kristali için k nokta sayısına karşılık hesaplanan toplam enerji değeri.

Tablo1. LiAlSi kristali için kesme enerjileri ve kesme enerjilerine karşılık gelen taban durum enerjileri.

\begin{tabular}{|c|c|c|c|c|c|}
\hline E $_{\text {cut }}$ (Ryd) & 20 & 40 & 60 & 80 & 100 \\
\hline E $_{\boldsymbol{0}}$ (Ryd) & -23.9881 & -23.9888 & -23.9892 & -23.9892 & -23.9892 \\
\hline
\end{tabular}

LiAlSi kristalinin yapısal ve elektronik parametrelerini belirlemek için k-noktaları 12x12x12 olarak kullanılmıştır. Şekil 3 de LiAlSi kristalinin k nokta sayısına bağlı olarak değişen toplam enerji değerleri görülmektedir. LiAlSi kristalinin kendinden tutarlı Kohn-Sham denklemlerinin (Kohn ve Sham, 1965) çözümleri elde edildikten sonra dinamik özellikleri yoğunluk fonksiyoneli pertürbasyon teorisi ile hesaplanmıştır. Brilloin bölgesinde 4x4x4'lük q-noktaları (Monkhorst ve Pack, 1965) ile LiAlSi kristali için dinamik matrisler belirlenmiştir. Bu hesaplamalar yapılırken $10^{-8}$ Ryd olacak şekilde gerçekleştirilerek hassas hesaplamaların yapılmasına özen gösterilmiştir.

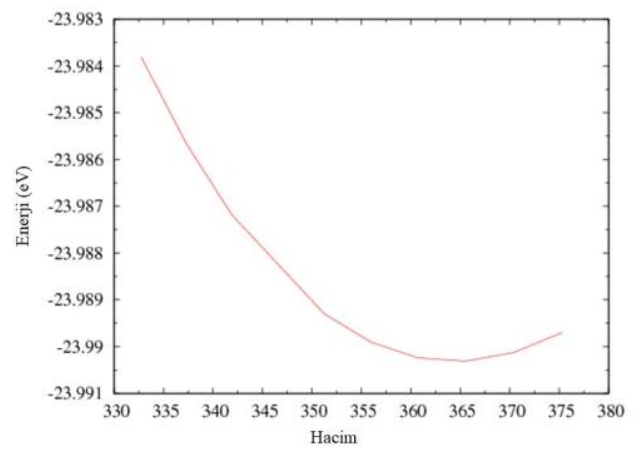

Şekil 4. LiAlSi kristali için hacim-toplam enerji bağlılığ 


\section{Araştırma Sonuçları ve Tartışma}

Bir malzemenin kararlılığı ve örgü parametresi (a $(\AA))$, yığın modülü (B (GPa)), yığın modülünün basınca göre birinci türevi $\left(B^{\prime}\right)$ gibi fiziksel özellikleri o malzemenin toplam enerjisi ile belirlenir. Bu sebeple LiAlSi kristalinin toplam enerjisi düzlem dalga psödopotansiyeller kullanılarak hacmin bir fonksiyonu olarak hesaplanmıştır. Şekil 4 de LiAlSi kristali için hacme bağlı toplam enerji değerleri çizilmiş̧ir. LiAlSi kristalinin hacmin bir fonksiyonu olarak toplam enerjileri genel gradyent yaklaşımı kullanılarak belirlenip örgü parametresi, yığın modülü ve yığın modülünün basınca göre birinci türevi aşağıdaki denklemde gösterilmiş olan BirchMurnaghan denklemine (Murnaghan, 1944) fit edilerek tespit edilmiştir. Elde edilen sonuçlar teorik ve deneysel sonuçlar ile uyum içerisindedir. Tablo 2'de bu sonuçlar sıralanmıştır.

$$
E(V)=E_{0}+V_{0} B_{0}\left(\frac{9}{16}\left(\left(\frac{V_{0}}{V}\right)^{\frac{2}{3}}-1\right)^{3} B_{0}^{\prime}+\frac{9}{16}\left(\left(\frac{V_{0}}{V}\right)^{\frac{2}{3}}-1\right)^{2}\left(6-4\left(\frac{V_{0}}{V}\right)^{\frac{2}{3}}\right)\right.
$$

Tablo 2. Hesaplanan örgü parametersi, yığın modülü ve yığın modülünün basinca göre birinci türevi.

\begin{tabular}{|l|l|c|}
\hline \multicolumn{1}{|c|}{ a $(\AA)$} & B (GPa) & $B^{\prime}$ \\
\hline 6.0306 [Bu çalışma] & 60.66 & 4.44 \\
\hline 5.9105 (Shan ve diğ., 2018) & 65.28 & 5.00 \\
\hline 5.9220 (Backelmann ve & & \\
Schuster, 1974) & & \\
\hline 5.9280 (Spina ve diğ., 2003) & & \\
\hline 5.9370 (Kandpal ve diğ., & & \\
2006) & & \\
\hline 6.0200 (Kacimi ve diğ., 2014) & & \\
\hline
\end{tabular}

Kristal yapılar için elektronik band yapısının bilinmesi önemlidir. Hesaplanan örgü sabiti kullanılarak LiAlSi kristalinin elektronik band yapısı incelenmiştir. Bu incelemede $\Gamma \rightarrow \mathrm{X} \rightarrow \mathrm{W} \rightarrow \mathrm{L} \rightarrow \Gamma \rightarrow \mathrm{K} \rightarrow \mathrm{W} \rightarrow \mathrm{U}$ yüksek simetri noktaları boyunca yapılmış olup, Brillouin bölgesinde $104 k$ noktası ile elektronik band yapısı incelenmiştir. Elde edilen sonuçlar gösterdi ki LiAlSi kristali $\mathrm{P}=0.0$ GPa basınç altında yarıiletken özelliği göstermektedir. Şekil 5 de LiAlSi kristalinin elektronik band yapısı görülmektedir. Elektronik band aralığ $0.40 \mathrm{eV}$ olup $\Gamma-\mathrm{X}$ indirekt band şeklindedir. Literatürdeki diğer teorik sonuçlar ile uyum içindedir. [1, 14] LiAlSi kristalinin elektronik band yapısı incelenecek olursa hem valans hem de iletim durumlarının dağılımı açık şekilde görülmektedir. Valans dağılımına bakıldığında $0.0 \mathrm{eV}$ ile yaklaşı $-6 \mathrm{eV}$ enerji aralığına lokalize olduğu, iletim band dağılımına bakıldığında $\Gamma$ noktasında $\sim 1.8 \mathrm{eV}, \mathrm{X}$ noktasında $\sim 0.5 \mathrm{eV}$ şeklinde lokalize olduğu görülmektedir. $\mathrm{P}=8.892 \mathrm{GPa}$ basınç uygulandığında ise elektronik band aralığ $-0.0082 \mathrm{eV}$ olduğu bulunmuştur. Sıfira çok çok yakın olan elektronik band aralığı yaklaşı $0.0 \mathrm{eV}$ kabul edilebilir. Böylelikle $\mathrm{P}=0.0 \mathrm{GPa}$ basınç altında yariletken özelliği gösteren LiAlSi kristali $\mathrm{P}=8.892 \mathrm{GPa}$ basınç altında iletken özelliği göstermektedir. $\mathrm{P}=8.892 \mathrm{GPa}$ basınç altında LiAlSi kristalinin elektronik band yapısı Şekil 6 de gösterilmiştir.

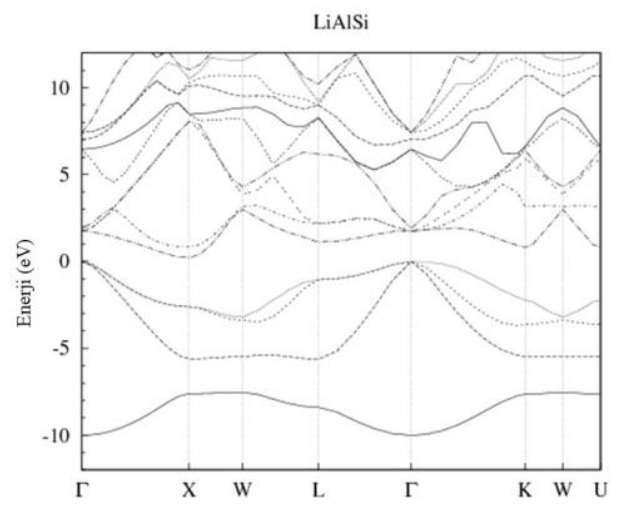

Şekil 5. LiAlSi kristalinin P=0.0 GPa basınç altında elektronik band yapısı. 


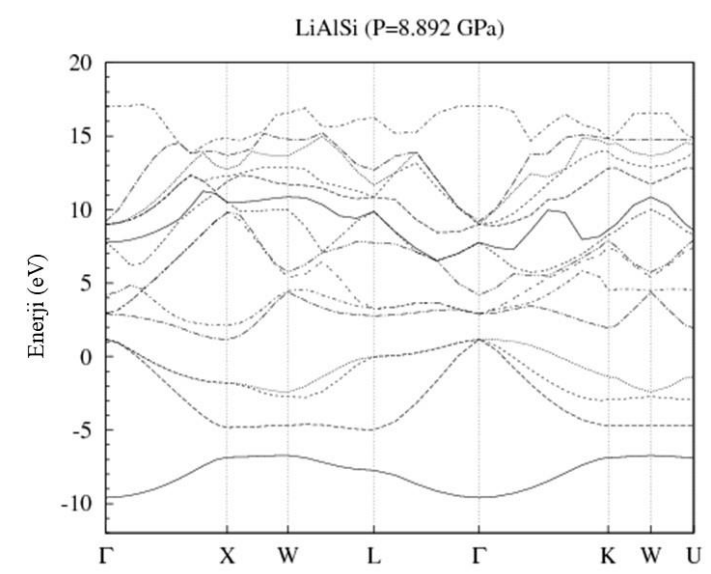

Şekil 6. LiAlSi kristalinin P=8.892 GPa basinç altında elektronik band yapısı.

Tablo 3. LiAlSi kristali için $P=0.0 \mathrm{GPa}$ ve $P=8.892 \mathrm{GPa}$ basınç altında enerji aralıkları.

\begin{tabular}{|c|c|c|c|}
\hline $\mathbf{P}(\mathbf{G P a})$ & $\begin{array}{c}\mathbf{E}_{\Gamma \cdot \Gamma} \\
(\mathrm{eV})\end{array}$ & $\begin{array}{c}\mathbf{E}_{\Gamma \cdot \mathbf{x}} \\
(\mathrm{eV})\end{array}$ & $\begin{array}{c}\mathbf{E}_{\Gamma \cdot \mathbf{L}} \\
(\mathrm{eV})\end{array}$ \\
\hline 0.0 & $\sim 1.8$ & $\sim 0.4$ & $\sim 1.5$ \\
\hline 8.892 & $\sim 2.0$ & $\sim 0.0$ & $\sim 1.9$ \\
\hline
\end{tabular}

İkinci olarak kübik yapıda ve $F \overline{4} 3 m$ uzay grubuna ait LiAlSi kristalinin fonon dağılım eğrisi ve fonon durum yoğunluğu incelenmiş̧tir. LiAlSi kristalinin birim hücresinde 3 atom bulunduğundan dolayı 9 adet fonon modu bulunmaktadır. Bunlardan 3'ü akustik mod ve 6 tanesi optik moddur. Akustik ve optik modlar, sırasıyla uzunlamasına akustik (LA), uzunlamasına optik (LO), enine akustik (TA) ve enine optik (TO) modlar olarak verilir. Şekil 7 da LiAlSi kristalinin $\mathrm{P}=0.0 \mathrm{GPa}$ basınç altındaki fonon dağılımı ve şekil 8 de fonon durum yoğunluğu verilmektedir. Fonon modlar 0 ile $575 \mathrm{~cm}^{-1}$ arasında dağıllım göstermektedir. Akustik ve optik modlar arasında bir aralık vardır. Ayrıca fonon frekans değerleri negatif değer almadığından LiAlSi kristalinin $\mathrm{P}=0.0 \mathrm{GPa}$ basınç altında kararlı olduğu ifade edilebilir. $8.892 \mathrm{GPa}$ değerinde basınç uygulandığında fonon dağılım eğrisi Şekil 9 da ve $8.892 \mathrm{GPa}$ basınç altındaki fonon durum yoğunluğu ise Şekil 10'da verilmiştir. Bu basınç altında elde edilen fonon dağılım eğrisi incelendiğinde fonon frekans değerlerinin pozitif değerler aldığı görülmektedir. Bu da P=8.892 GPa basınç altında da LiAlSi kristalinin kararlı olduğu bilgisinin verir. Yani LiAlSi kristali 8.892 GPa basınç altında kararlılı̆g korunmakta ancak elektronik özellikleri değişmektedir.

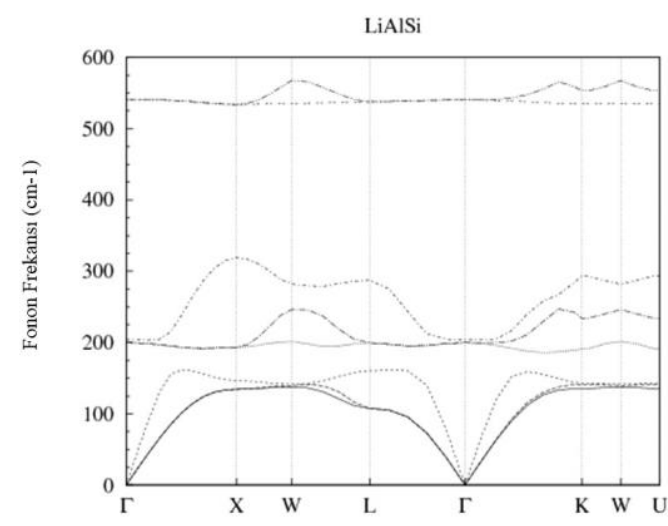

Şekil 7. LiAlSi kristalinin P=0.0 GPa basinç altında fonon dă̆gllım ĕgrisi. 


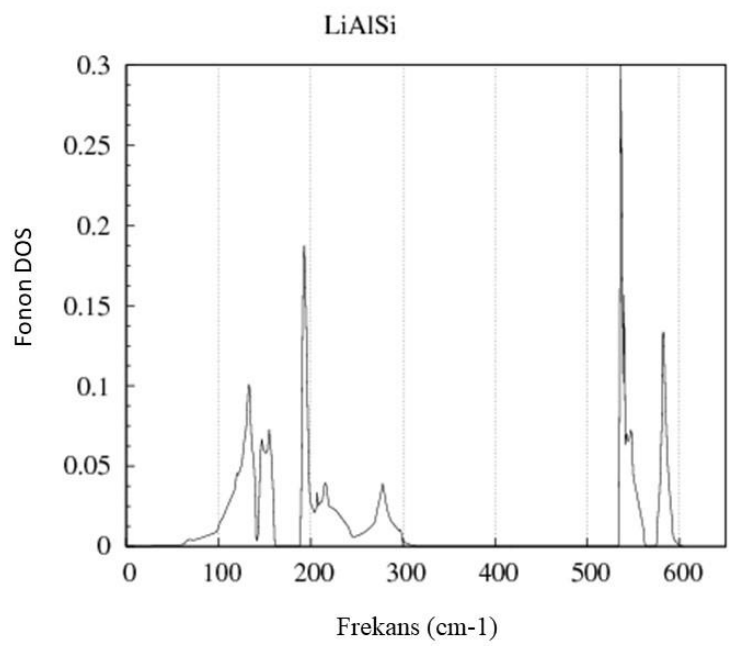

Şekil 8. LiAlSi kristalinin P=0.0 GPa basınç altında fonon durum yoğunluğu.

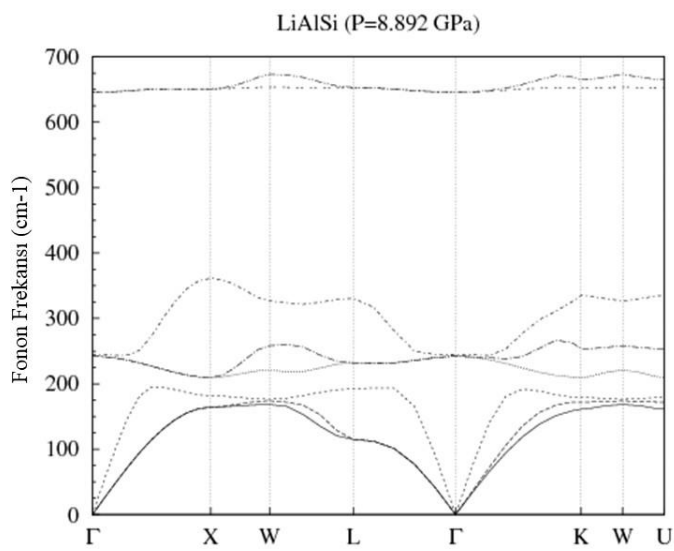

Şekil 9. LiAlSi kristalinin P=8.892 GPa basınç altında fonon dă̆glım ĕgrisi.

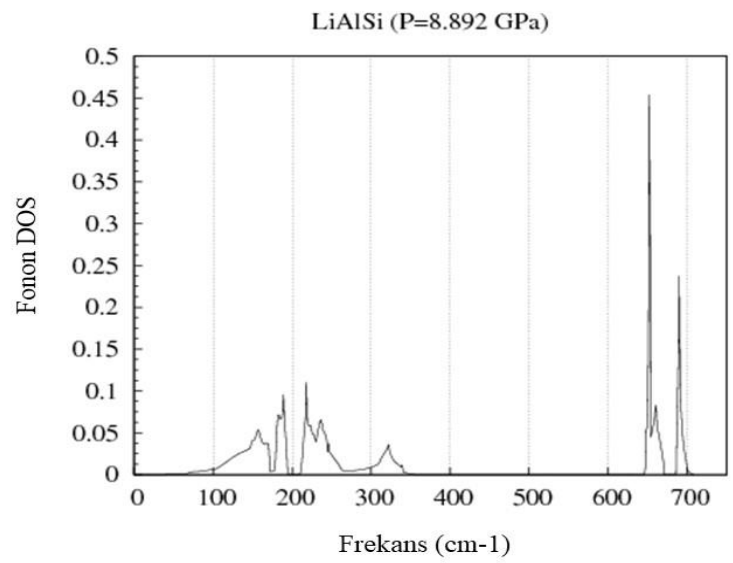

Şekil 10. LiAlSi kristalinin P=8.892 GPa basınç altında fonon durum yoğunluğu. 


\section{Sonuç}

Bu çalışmada LiAlSi kristalinin yapısal, elektronik ve titreşim özellikleri incelenmiş̧ir. Quantum Espresso programı kullanılarak Yoğunluk Fonksiyoneli Teorisi Genel Gradyent Yaklaşımı ile örgü parametresi, yığın modülü ve yığın modülünün basınca gore birinci türevi hesaplanmıştır. Daha sonra elektronik band aralığı hesaplanarak LiAlSi kristalinin $\mathrm{P}=0.0 \mathrm{GPa}$ basınç altında yarıiletken özelliği gösterdiği bulunmuştur. $\mathrm{P}=8.892 \mathrm{GPa}$ basınç uygulandığında ise LiAlSi kristali iletken özelliği gösterdiği ortaya konmuştur. $\mathrm{P}=0.0$ GPa ve $\mathrm{P}=8.892 \mathrm{GPa}$ basınç değerlerindeki fonon dağılım eğrileri elde edilmiş̧ir. Elde edilen bu bulguları literatürde karşılaştıracak çalışmalar bulunmamaktadır. Bu çalışma bulgularının geleceğe ışık tutacağı düşünülmektedir.

\section{Kaynakça}

Kandpal, H. C., Felser, C., Seshadri, R. (2006). Covalent bonding and the nature of band gaps in some half-Heusler compounds. J. Phys. D.:Appl. Phys. 39, 776-785. https://doi.org/10.1088/0022-3727/39/5/S02

Casper, F., Grof, T., Chadov, S., Balke, B., Felser, C. (2012). Half-Heusler compounds: novel materials for energy and spintronic applications. Semicond. Sci. Technol. 27, 063001/1-8. https://doi.org/10.1088/0268-1242/27/6/063001

Boom, E. (1949). Doklady Akademii Nauk SSSR 645-646.

Tillard, M., Belin, C.,Spina, L., Jia, Y. Z. (2005). Phase stabilities electronic and electrochemical properties of compounds in the LiAl-Si system. Solid States Science 7, 1125-1134. https://doi.org/10.1016/j.solidstatesciences.2005.04.010

Barth, J., Fecher, G. H., Schwind, M., Beleanu, A., Felser, C., Shkabko, A., Weidenkaff, A., Hass, J., Reller, A., Köhne, M. (2010). Investigation of the Thermoelectric Properties of LiAl Si and LiAlGe. Journal of Electronic Materials, 39(9), 1856-1860. DOI: $10.1007 / \mathrm{s} 11664-010-1076-9$

Nowotny, H., Holub, F. (1960). Untersuchungen an metallischen Systemen mit Flußspatphasen. Mon. Für Chem. Verwandte-.Teil. und Wiss. 91, 877-887. https://doi.org/10.1007/BF00929560

Spina, I., Jia, Y. Z., Ducourant, B., Tillard, M., Belin, C. (2003). Optoelectronic and transport properties of LiBZ (B= Al, In, Ga and $\mathrm{Z}=\mathrm{Si}, \mathrm{Ge}, \mathrm{Sn}$ ) semiconductors. Z. Für Krist.-Cryst. Matter, 218, 740-746. https://doi.org/10.1016/j.jssc.2017.12.014

Schuster, H. U., Hinterkeuser, H. W., Schafer, W., Will, G.. (1976). Investigation on Neutron Diffraction of the Phases LiAlSi and LiAlGe, Z. Naturforsch. 316, 1540-1541.

Baroni, S., Corso, A. D., de Gironcoli, S., Giannozzi P. http://www.pwscf.org

Perdew, J. P., Burke, K., Ernzerhof, M. (1996). Generalized gradient approximation made simple. Physical Review Letters, 77, $3865-$ 3868. DOI: https://doi.org/10.1103/PhysRevLett.77.3865

Kohn, W., Sham, L. J. (1965). Self-Consistent Equations Including Exchange and Correlation Effects. Physical Review, 140, 11331138. DOI: https://doi.org/10.1103/PhysRev.140.A1133

Monkhorst, H. J., Pack, J. D. (1976). Special points for Brillouin-zone integrations. Physical Review B, 13, 5188-5192. DOI: https://doi.org/10.1103/PhysRevB.13.5188

Murnaghan, F. D. (1944). The compressibility of media under extreme pressure. Proceedings of the National Academy of Sciences of the United States of America, vol. 30(9), 244-247. doi: 10.1073 / pnas.30.9.244

Shan, S. H., Khan, S. H., Lafer, A., Murtaza, G.. (2018). Optoelectronic and transport properties of LiBZ (B: Al, In, Ga and Z: Si, Ge, Sn) semiconductors. Journal of Solid State Chemistry 258, 800-808. https://doi.org/10.1016/j.jssc.2017.12.014

Backelmann, W., Schuster, H. U. (1974). Ternäre Phasen im Dreistoffsystem Lithium - Gallium - Germanium. Z. Für Anorg. Allg. Chem. 410, 233-240. https://doi.org/10.1002/zaac.19744100303

Kacimi, S., Mehnane, H., Zaoui, A. (2014). I-II-V and I-III-IV half-Heusler compounds for optoelectronic applications: Comparative ab initio study. J. Alloy. Compd. 587, 451-458. https://doi.org/10.1016/j.jallcom.2013.10.046 